\title{
The Follicular Output Rate (FORT) as a method to evaluate transdermal testosterone efficacy in poor responders
}

\author{
Roser Solernou ${ }^{1}$, Sara Peralta ${ }^{1}$, Gemma Casals ${ }^{1}$, Marta Guimera ${ }^{1}$, Marina Solsona ${ }^{1}$, Aina Borras ${ }^{1}$, Dolores \\ Manau $^{1}$, Francesc Fàbregues ${ }^{1}$
}

${ }^{1}$ Institute Clinic of Gynecology, Obstetrics and Gynecology. Hospital Clinic. Barcelona, Spain

\begin{abstract}
Objective: Follicular Output Rate (FORT) is an efficient quantitative and qualitative marker of ovarian responsiveness to gonadotropins. Transdermal testosterone (TT) has been used as adjuvant therapy to gonadotrophins in order to improve ovarian response in poor responders (PR). The aim of this study was to analyze whether TT can improve follicular sensitivity to gonadotropins using FORT.

Methods: This retrospective study, held in a tertiarycare university hospital included 90 PR patients, according to the Bologna criteria. Patients in Group $1(n=46)$ received transdermal application of testosterone preceding gonadotrophin ovarian stimulation under pituitary suppression. In Group $2(n=44)$ ovarian stimulation was carried out with high-dose gonadotrophin in association with minidose $\mathrm{GnRH}$ agonist protocol. We analyzed ovarian stimulation parameters and IVF outcomes. We determined antral follicle count (AFC) (3-8 mm) before ovarian stimulation, pre-ovulatory follicle count (PFC) $(16-22 \mathrm{~mm})$ and the day of hCG administration. We calculated the FORT using the PFCx100/AFC ratio.

Results: Baseline characteristics and ovarian reserve parameters were similar in both groups. FORT and oocytes retrieved were significantly higher in group 1 vs group 2 . There were no significant differences in pregnancy rates. In group 1 there was a significant correlation between FORT and AFC.

Conclusions: This study suggests that the potential beneficial mechanism of $T T$ in poor responder patients may be based on increasing the antral follicle sensitivity to gonadotrophin. FORT is an excellent tool to demonstrate this.
\end{abstract}

Keywords: Follicular Output Rate, Transdermal testosterone, Poor responder, ovarian stimulation

\section{INTRODUCTION}

The management of poor responders (PR) is a major challenge in IVF. Various stimulation regimens and interventions have been proposed for improving pregnancy outcomes in PR. These also include several adjuvant therapies such as androgen supplements (dehidroepiandrosterone and testosterone) and GH (Ubaldi et al. 2014, Ata \& Seli, 2015, Kamath et al., 2019). Most of the Randomized Clinical Trials (RCT) evaluating these clinical add-ons had poorly reported methodologies, small sample sizes, or were at risk of bias. The conclusions for almost all the clinical adjuncts is the need for robust, welldesigned RCTs to address both clinical and cost-effective parameters of the interventions. Although all of these adjuvant therapies provided promising results in initial studies, their efficacy has not been solidly demonstrated in RCT studies. The challenge in conducting an adequately powered RCT is the sample size. To show an improvement of $5 \%$ in LBR with $80 \%$ power after an intervention, a sample size of more than 2,000 participants is needed, which would be difficult to recruit even in large centers (Kamath et al., 2019).

According to results obtained in animals, it is possible that androgens exert a facilitating role in follicle responsiveness to $\mathrm{FSH}$, which leads to an increase in the number of growing follicles (Weil et al., 1999; Vendola et al., 1998; 1999). A simple method to evaluate these effects is controlled ovarian hyperstimulation $(\mathrm{COH})$; however, ovarian response to $\mathrm{COH}$ is quantitatively influenced by the pretreatment amount of small antral follicles.

To overcome this difficulty, an objective index to assessing antral follicle responsiveness to exogenous $\mathrm{FSH}$, the Follicular Output Rate (FORT) is being developed (Genro et al., 2011; Gallot et al., 2012; Grynberg \& Labrosse, 2019). FORT is calculated based on the ratio between the number of preovulatory follicles obtained in response to $\mathrm{FSH}$ administration and the preexisting pool of small antral follicles. Hence, in case of androgen pretreatments, FORT constitutes an interesting clinical strategy to check whether the ability of antral follicles to respond to exogenous gonadotrophins has been improved (Fanchin et al., 2011).

The aim of this study was precisely to assess whether transdermal testosterone was able to improve FORT in poor responders.

\section{MATERIALS AND METHODS}

\section{Patients}

This study was performed by a retrospective analysis of our database of women referred to our center for IVF, and was conducted from January 2013 to May 2016 in the Assisted Reproduction Unit of the Hospital Clinic in Barcelona (Spain). Before starting the IVF cycle, the patients were suggested to use Transdermal testosterone (TT) as an adjuvant therapy to ovarian stimulation. Two patients rejected this therapy. Review Board and informed consent was obtained from all individual participants, included in the study (HB-15-EL-RS-C). We recruited 90 poor responder patients according to the Bologna criteria (Ferraretti et al., 2011). All the patients were in good normal thyroid, kidney and hepatic health laboratory results, and they had regular menstruation periods with a 21-35 days duration. None of them had taken any infertility medication in the 3 months prior to the study.

We used the long GnRh agonist protocol in all the patients. Group 1 patients were treated with $T$ preceding ovarian stimulation with gonadotropins, and Group 2 started ovarian stimulation after ovarian arrest was confirmed. (Fig 1).

FORT was calculated as the ratio of pre-ovulatory follicle count (PFC)(16-22 $\mathrm{mm}$ in diameter) on dhCG $x$ $100 /$ small antral follicle (3-8 $\mathrm{mm}$ in diameter) count 


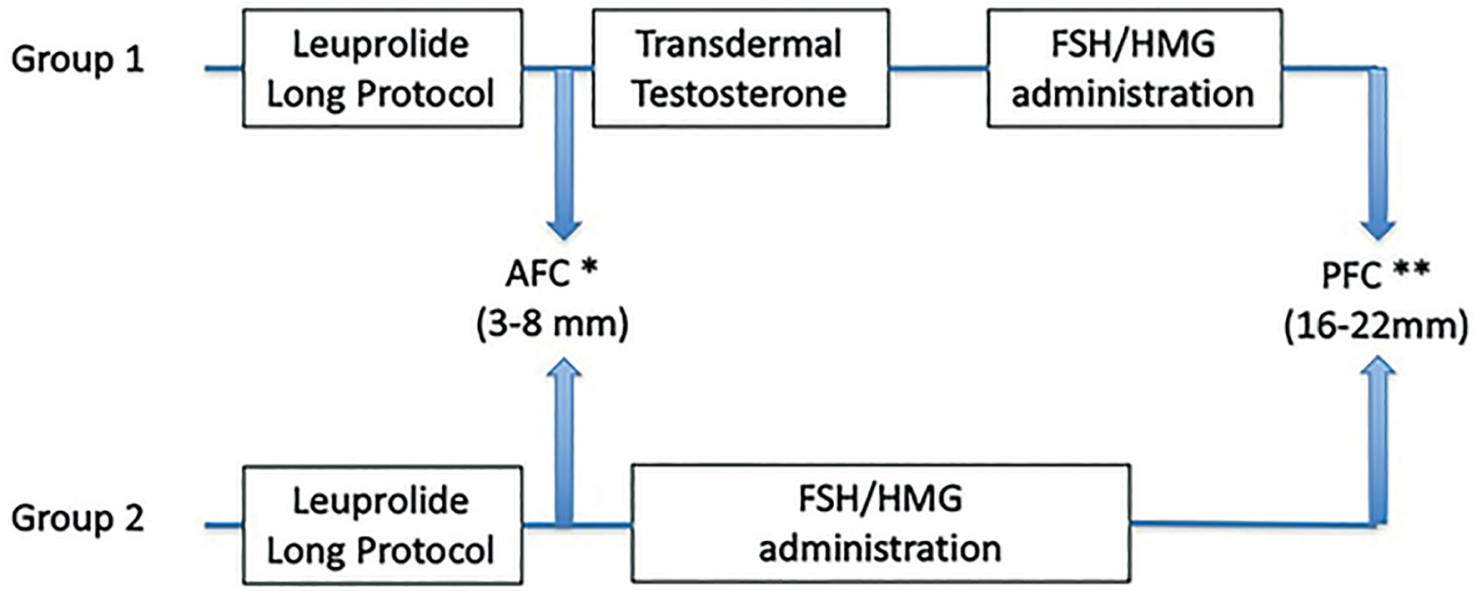

AFC*: Antral follicle count; PFC*: Preovulatory follicle count

Figure 1. Schematic representation protocol

at baseline (ovarian arrest day) (Fig 2). We evaluated the following study parameters: days of stimulation, pre-ovulatory follicles on the day of human chorionic gonadotropin (hCG) administration, number of oocytes retrieved, number of embryos achieved and transferred. We also assessed pregnancy outcomes, including clinical and ongoing pregnancy rates. cycles

There was no perimplantation diagnosis in any of the

\section{Stimulation regimens}

We suppressed the pituitary suppression by S.C administration of leprolide acetate (Procrin; Abbott Laboratories, Madrid, Spain). This treatment was started in the midluteal phase of the previous cycle and given 1 $\mathrm{mg}$ daily, then reduced to $0.5 \mathrm{mg}$ after ovarian arrest was confirmed, and continued until the administration of HCG.

Group-1 patients started $T T$ on the day when pituitary suppression was confirmed and the therapy was continued for 5 days. Transdermal testosterone treatment was carried out using a daily single patch with a $2.5 \mathrm{mg} /$ day nominal delivery rate of testosterone (Testopatch, Pierre Fabre Iberica SA, Barcelona, Spain), which was applied to the thigh at night and removed always at 09:00h in the morning.

This transdermal delivery system maintains testosterone levels stable within narrow ranges with little within - and between - subject variation, providing a highly controllable and reliable way of delivering testosterone, and the hormonal dose administered can be modified according to the patch duration (Buckler et al., 1998; De Sanctis et al., 1998; Mazer, 2000). We chose to use $20 \mathrm{mg} /$ $\mathrm{kg}$ of testosterone per day for 5 days, based on previous experimental studies in primates (Vendola et al., 1998; 1999). Thus, in each patient, the patch was applied at night, supposedly left in place for a predetermined number of hours in order to provide the desired daily dose of testosterone (e.g. in a woman weighing $60 \mathrm{~kg}$ and needing $1,200 \mathrm{mg} /$ day, the patch was used for $12 \mathrm{~h}[0.1 \mathrm{mg} / \mathrm{h}$ delivery rate $12 \mathrm{~h} .1 .2 \mathrm{mg}$ or $1200 \mathrm{mg}$ ], and applied at $21: 00 \mathrm{~h}$ ). We performed the testosterone therapy according to a routinely used protocol (Balasch et al., 2006; Fàbregues et al., 2009; 2019).

In Group 2 gonadotropin therapy started after ovarian arrest was confirmed. In both groups controlled ovarian hyperstimulation was performed with 300IU per day of r-hFSH (Gonal-F, Merck S.A., Madrid, Spain) together with 75IU HMG (Menopur, Ferring S.A., Madrid, Spain). The criteria for hCG administration (250mg s.c.Ovitrelle, Serono S.A.) were the presence of two or more follicles $>18 \mathrm{~mm}$ in diameter, with $>4$ follicles measuring $>14 \mathrm{~mm}$ in association with a consistent rise in serum E2 concentration.

The cycle was cancelled when there were less than 3 follicles with diameter $>14 \mathrm{~mm}$ after 8-9 days of gonadotropin therapy, or after 4-5 additional treatment days without attaining, or the imminent prospect of attaining, the criteria for hCG administration.

Oocyte aspiration was performed under vaginal ultrasonography 35-36h after hCG administration. Embryo grading was recorded according to published criteria (Veeck, 1999); embryos graded 1 or 2 were considered of high quality. In both groups, embryo transfer was performed in the cleavage stage (day 3 ). The luteal phase was supported with vaginal micronized progesterone $(600 \mathrm{mg} /$ day given at $8 \mathrm{~h}$ intervals) starting on the day following oocyte aspiration and continuing either up to menstruation or, if the patients became pregnant, for at least the first 3 weeks of pregnancy.

Pregnancy was diagnosed by a positive serum $\beta$-hCG test 12 days after ET. Clinical pregnancy was defined by observation of a fetal heartbeat using transvaginal ultrasonography upon 5-6 weeks of gestation.

\section{Statistical analysis}

Sample size was based on two previous studies from our group, showing that: (i) multifollicular development and oocyte retrieval was obtained in $80 \%$ of previously low responders receiving pretreatment with $\Pi T$ and gonadotrophin ovarian stimulation as used in patients in Group 1 (Balasch et al., 2006); and (ii) 46\% of patients having their first cycle of IVF cancelled because of poor follicular response underwent oocyte retrieval in the second IVF attempt, when treated according to the approach used in Group 2 (Peñarrubia et al; 2005). The sample size required to provide an $80 \%$ power to detect this magnitude of treatment effect between groups was calculated to be a minimum of 31 patients per group, using a two-tailed analysis with a detection limit of $5 \%$, to avoid a type I error in hypothesis testing. 


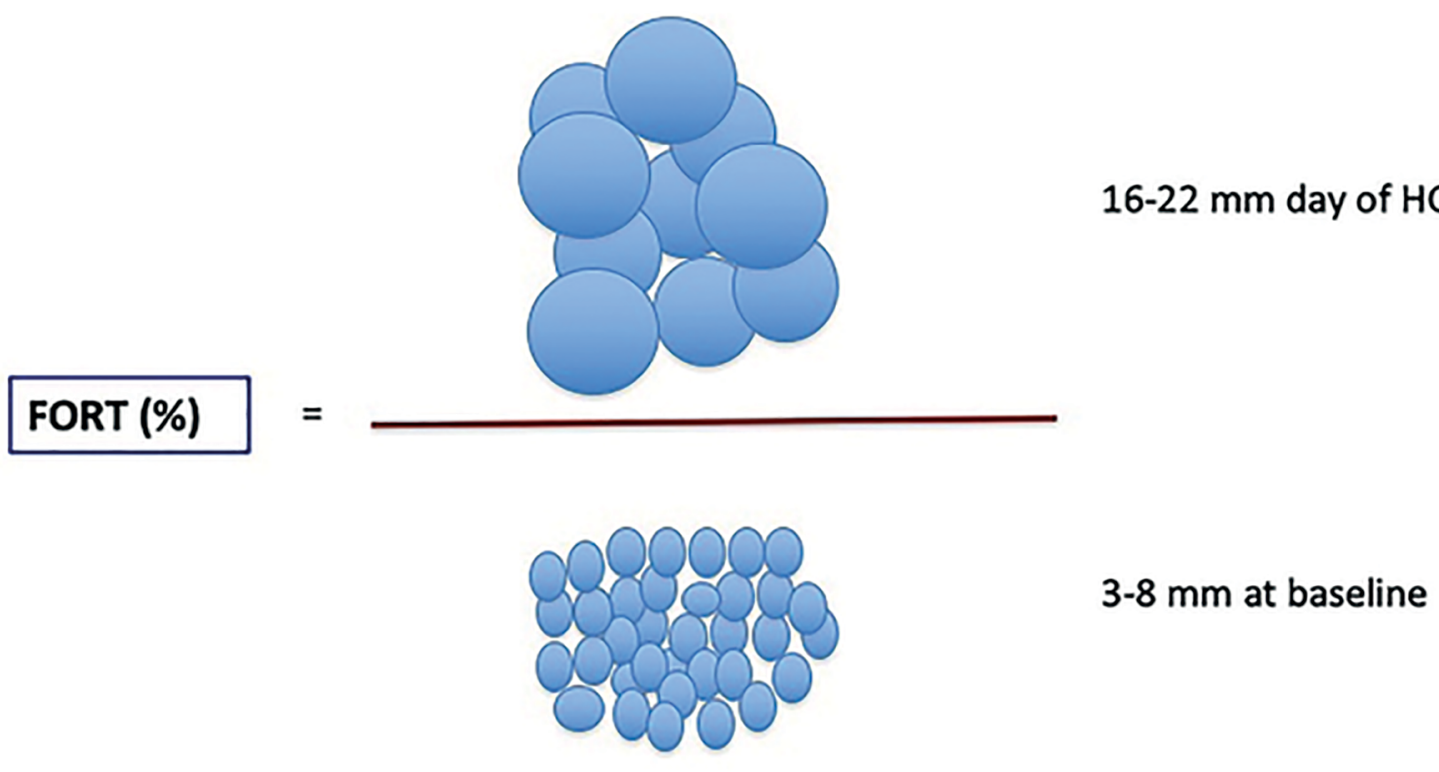

Figure 2. Follicular output rate $=$ Ratio of pre-ovulatory follicle count on day hCG $\times 100 /$ antral follicle count

All statistical analyses were performed using the SPSS version 23.0 software (Chicago, IL, USA). We used a t-test to compare the mean values between two different stimulation protocols. Differences in outcome rates were analyzed using an $\mathrm{X} 2$ test or the Fisher's exact test. $\mathrm{p}<0.05$ was considered statistically significant. The Spearman's test was used to determine the correlation between FORT and ovarian reserve parameters in patients treated with TT.

\section{RESULTS}

As summarized in Table 1 the groups were similar with respect to age, body mass index (BMI), duration of infertility, infertility causes, antral follicle count (AFC), Anti-Müllerian hormone (AMH) levels and basal FSH and estradiol. There were no reported major side effects after testosterone therapy, and two protocols were well tolerated by all patients.

Table 2 shows stimulation parameters and IVF outcomes, and we found that the number of cancelled cycles was similar in both groups (10.9 vs $22.8 \%$ ). The duration of ovarian stimulation was significantly shorter in Group 1 when compared with Group 2 (10.2 \pm 0.3 vs $11.5 \pm 0.2$ days). FORT and number of oocytes retrieved were also significantly higher in Group 1 vs Group 2 ( $73 \pm 4.6$ vs $55.6 \pm 4.1 \%)$; and ( $4 \pm 0.3$ vs $3 \pm 0.3)$, respectively. There were no differences in fertilization, implantation and pregnancy rates in both groups.

When we evaluate the correlations between FORT and ovarian reserve parameters in Group-1 patients, there was a significant correlation with $\operatorname{AFC}(r=0.41 ; p=0.001)$. However, this was not demonstrated with age, basal FSH and $\mathrm{AMH}$ levels.

\section{DISCUSSION}

To our knowledge, this is the first study to analyze the value of FORT in poor responder patients, and specifically in those who have undergone adjuvant treatment with $T$ in IVF cycles.

The Cochrane review on the role of testosterone pretreatment included four RCTs with poor responders (Nagels et al., 2015). Pooling the RCTs showed that pretreatment with testosterone was associated with improved LBR (OR 2.60; 95\% CI, 1.30-5.20; 4 RCTs, $\mathrm{n}=345$ ). However, a sensitivity analysis removing the studies at high risk of performance bias showed no statistically significant difference in LBR between the testosterone and control groups (OR 2.00; 95\% CI, 0.17$23.49 ; 1 \mathrm{RCT}, \mathrm{n}=53$ ). These contradictory results could be explained by the different doses used (10 to $12 \mathrm{mg} /$ daily), the way of application (patches or gel) and the pretreatment length (5 to 21 days) (Massin et al., 2006; Fàbregues et al., 2009;2019; Kim et al., 2011; Doan et al., 2017). Obviously, only a multicenter randomized study with a large number of patients can definitively clarify the true efficacy of this add-on therapy in ovarian stimulation (Polyzos et al., 2018).

The above notwithstanding, in this study we chose to use $\pi$ for 5 days on the basis of studies in primates (Vendola et al., 1999), and also reports from previous clinical studies (Balasch et al., 2006; Fàbregues et al., $2009 ; 2013 ; 2019)$. The effects of $\mathrm{T}$ on follicular response appears to be mediated by increasing FSH-receptor activity and by stimulating insulin-like growth factor I (IGF-I) (Weil et al.,1999). In studies in subhuman primates, androgen receptor gene expression correlated with follicle growth, and the $\mathrm{T}$ treatment significantly increased granulosa cell (GC) FSH receptor messenger RNA (mRNA) (Vendola et al., 1998). In this line, previous studies have shown that just 5 days of $T$ caused a progressively increased level of circulating IGF-I throughout the ovarian stimulation for more than a week after $\Pi$ was discontinued, and it has been suggested that increased levels of IgF1 could amplify the effect of gonadotropins in the ovary (Balasch et al., 2006; Meldrum et al., 2013).

Regardless of the controversy that exists regarding the clinical evidence of the androgen therapy usefulness, it is especially interesting to analyze whether there really is a greater capacity of androgens for the sensitization of follicles to the action of gonadotropins. In this sense, FORT is an excellent method to evaluate it (Fanchin et al., 2011).

Interestingly, the number of pre-ovulatory follicles obtained at the end of controlled ovarian hyperstimulation $(\mathrm{COH})$ is not a reliable reflection of antral follicle sensitivity to $\mathrm{FSH}$, as it is greatly influenced by the number of the 


\begin{tabular}{|c|c|c|}
\hline Variable & $\begin{array}{c}\text { Group 1 } \\
(n=46)\end{array}$ & $\begin{array}{c}\text { Group } 2 \\
(n=44)\end{array}$ \\
\hline Age (years) & $36.8 \pm 0.3$ & $37 \pm 0.3$ \\
\hline BMI $\left(\mathrm{Kg} / \mathbf{m}^{2}\right)$ & $24 \pm 0.5$ & $24.2 \pm 0.7$ \\
\hline $\begin{array}{l}\text { Infertility factor } \\
\text { Male factor }(\mathbf{n}, \%) \\
\text { Unexplained }(\mathbf{n}, \%) \\
\text { Tubal factor }(\mathbf{n}, \%) \\
\text { Endometriosis }(\mathbf{n}, \%)\end{array}$ & $\begin{array}{c}21(45.6) \\
11(23.9) \\
9(19.5) \\
5(10.8)\end{array}$ & $\begin{array}{c}18(40.9) \\
10(22.7) \\
10(22.7) \\
6(13.6) \\
\end{array}$ \\
\hline Duration of infertility (years) & $5.8 \pm 2.7$ & $5.6 \pm 2.2$ \\
\hline Basal FSH (IU/I) & $12.3 \pm 0.5$ & $13.2 \pm 0.2$ \\
\hline Antral follicle count ( $n$ ) & $5.4 \pm 0.4$ & $5.8 \pm 0.3$ \\
\hline AMH (ng/ml) & $0.7 \pm 0.3$ & $0.9 \pm 0.1$ \\
\hline
\end{tabular}

Values are mean \pm SD. Between group differences all non-significant at a 0.05 level of significance.

\begin{tabular}{|c|c|c|c|}
\hline Variable & Group 1 & Group 2 & p-value \\
\hline Patients with hCG and ovum retrieval $(n, \%)$ & $41(89.1)$ & $34(77.2)$ & 0.3 \\
\hline Days of ovarian stimulation & $10.2 \pm 0.3$ & $11.5 \pm 0.2$ & 0.01 \\
\hline Pre-ovulatory follicle $(16-22 \mathrm{~mm})$ & $4.1 \pm 1.2$ & $3 \pm 0.9$ & 0.2 \\
\hline FORT (\%) & $73 \pm 4.6$ & $55.6 \pm 4.1$ & 0.01 \\
\hline No. of oocytes retrieved ${ }^{a}$ & $4 \pm 0.3$ & $3 \pm 0.3$ & 0.04 \\
\hline No. of metaphase II oocytes ${ }^{a}$ & $4 \pm 0.3$ & $2.8 \pm 1.8$ & 0.15 \\
\hline No. of $2 \mathrm{PN}$ on day $1^{\mathrm{a}}$ & $3.9 \pm 0.1$ & $2.4 \pm 0.1$ & 0.1 \\
\hline No. of embryos obtaineda & $2.6 \pm 0.3$ & $1.7 \pm 0.1$ & 0.29 \\
\hline No of embryos transferreda & $1.7 \pm 0.5$ & $1.4 \pm 0.4$ & 0.28 \\
\hline Implantation rate $(\%)^{a}$ & 28.3 & 22.8 & 0.58 \\
\hline $\begin{array}{l}\text { Clinical pregnancy } \\
\text { Number } \\
\text { Per oocyte retrieval (\%) } \\
\text { Twins }(\mathrm{n}, \%) \\
\text { Miscarriages }(\mathrm{n}, \%)\end{array}$ & $\begin{array}{c}15 \\
36.5 \\
1 \\
1\end{array}$ & $\begin{array}{c}10 \\
29.4 \\
0 \\
1\end{array}$ & $\begin{array}{l}\text { N.S } \\
\text { N.S } \\
\text { N.S } \\
\text { N.S }\end{array}$ \\
\hline
\end{tabular}

Values are mean \pm SD. ${ }^{a}$ Values are relative to the number of patients with oocyte retrieval.

small antral follicles available before treatment. This contingency constitutes a possible explanation for the inconstant relationship between the absolute counting of growing follicles obtained in $\mathrm{COH}$ and IVF-ET outcomes (Fanchin et al., 2011; Grynberg \& Labrosse, 2019; Besow et al., 2019).

Several studies have established FORT groups according to tercile values: low $(<42 \%)$, average (42$58 \%$ ) and high ( $>58 \%$ ) in order to analyze the effective response to FSH and ovarian follicular competence (Gallot et al., 2012; Tan et al., 2019). According to the data from this study, the observed FORT can be considered mediumhigh ( $73 \pm 4.6$ vs $55.6 \pm 4.1)$, and this is in agreement with previous studies in the sense that with ovarian aging, antral follicles do not lose their aptitude to respond to $\mathrm{FSH}$, and probably reveals a compensating mechanism for preserving ovulatory folliculogenesis. (Gallot et al.,2012).

Taking into account the homogeneity of the studied groups, the significantly higher FORT in Group 1 vs 2 of the study has special relevance. This could also be related to a shorter duration on the days of stimulation and an increase in the number of oocytes obtained. This data agrees with those obtained in other studies (Gallot et al., 2012; Griesinger et al., 2019).

In accordance with previous studies, a correlation between FORT and antral follicle count was also shown in our study (Gallot et al., 2012); however, with AMH levels we did not find an aspect that was reported in other studies (Genro et al., 2011). It has been speculated that the negative correlation between AMH levels with FORT could be related to the possible inhibitory effect of this hormone with the sensitivity of follicles to FSH. However, this aspect has been suggested in patients with normal ovarian reserve but not in low responders.

$\Pi T$ has been used in poor responders with long $\mathrm{GnRH}$ agonist and antagonist protocols, finding no differences in terms of pregnancy rate or live birth rate (Fábregues et al., 2019). In this study, we included patients in whom the long agonist protocol was used based on the study sample size being calculated for this protocol.

The main limitation of this study was its retrospective design and small sample size. However, the poor responder 
population according to the Bologna criteria represents only a 5 to $10 \%$ of patients in most assisted reproduction clinics, which creates logistic problems when performing a prospective study with sufficient power. Although the patients were not randomized, the two populations had similar baseline characteristics, which made it possible to compare IVF outcomes between the groups.

Obviously, the findings of this study should be confirmed by other groups in larger randomized trials, which could shed light on the specific aspect of the sensitizing effect of $\pi$ pre-treatment in $\mathrm{COH}$ of poor responder patients according to the Bologna criteria.

\section{CONCLUSIONS}

Despite moderate evidence regarding the efficacy of $\Pi \pi$ as an adjuvant treatment in ovarian stimulation, this study suggests that the potential beneficial mechanism of $\Pi T$ in poor responder patients may be based on increasing the antral follicle sensitivity to the action of gonadotrophin treatment. FORT is an excellent tool to demonstrate this.

\section{CONFLICT OF INTEREST}

The author has no conflict of interest to declare.

\section{Corresponding Author:}

Francesc Fàbregues

Institut Clinic of Gynecology, Obstetrics and Gynecology

Hospital Clinic

Barcelona. Spain

E-mail: fgasol@clinic.cat

\section{REFERENCES}

Ata B, Seli E. Strategies for controlled ovarian stimulation in the setting of ovarian aging. Semin Reprod Med. 2015;33:436-48. PMID: 26562286 DOI: 10.1055/s-00351567818

Balasch J, Fábregues $F$, Peñarrubia J, Carmona $F$, Casamitjana R, Creus M, Manau D, Casals G, Vanrell JA. Pretreatment with transdermal testosterone may improve ovarian response to gonadotrophins in poor-responder IVF patients with normal basal concentrations of $\mathrm{FSH}$. Hum Reprod. 2006;21:1884-93. PMID: 16517559 DOI: 10.1093/humrep/del052

Besow C, Donato R, Souza T, Chajou R, Genro V, CunhaFilho JS. Antral follicle responsiveness assed by folicular output Rate (FORT) correlates with follicles diameter. J Ovarian Res. 2019;12:48. PMID: 31128593 DOI: 10.1186/ s13048-019-0522-4

Buckler HM, Robertson WR, Wu FC. Which androgen replacement therapy for women? J Clin Endocrinol Metab. 1998;83:3920-4. PMID: 9814469 DOI: 10.1210/ jcem.83.11.5280

De Sanctis V, Vullo C, Urso L, Rigolin F, Cavallini A, Caramelli K, Daugherty C, Mazer N. Clinical experience using the Androderm testosterone transdermal system in hypogonadal adolescents and young men with beta-thalassemia major. J Pediatric Endocrinol Metab. 1998;11:891-900. PMID: 10091163

Doan HT, Quan LH, Nguyen TT. The effectiveness of transdermal testosterone gel $1 \%$ (androgel) for poor responders undergoing in vitro fertilization. Gynecol Endocrionol. 2017;33:977-9. PMID: 28562099 DOI: $10.1080 / 09513590.2017 .1332586$
Fàbregues F, Peñarrubia J, Creus M, Manau D, Casals G, Carmona F, Balasch J. Transdermal testosterone may improve ovarian response to gonadotrophins in lowresponder IVF patients: a randomized, clinical trial. Hum Reprod. 2009;24:349-59. PMID: 19054777 DOI: 10.1093/ humrep/den 428

Fàbregues F, Iraola A, Casals G, Creus M, Peralta S, Peñarrubia J, Manau D, Civico S, Balasch J. IVF results following transdermal testosterone in poor responders according Bologna criteria. Hum Reprod. 2013;28:i311-56.

Fàbregues F, Solernou R, Ferreri J, Guimerá M, Peralta S, Casals G, Peñarrubia J, Creus M, Manau D. Comparison of $\mathrm{GnRh}$ agonist versus luteal estradiol GnRH antagonist protocol using transdermal tetosterone in poor responders. JBRA Assist Reprod. 2019;23:130-6. PMID: 30614665 DOI: $10.5935 / 1518-0557.20180090$

Fanchin R, Frydman N, Even M, Berwanger AL, Gynberg $M$, Ayoubi JM. Androgens and poor responders: are we ready to take the plunge into clinical theraphy? Fertil Steril. 2011;96:1062-5. PMID: 22036049 DOI: 10.1016/j. fertnstert.2011.09.050

Ferraretti AP, La Marca A, Fauser BC, Tarlatzis B, Nargund G, Gianaroli L; ESHRE Working Group on Poor Ovarian Response Definition. ESHRE consensus on the definition of 'poor response' to ovarian stimulation for in vitro fertilization: the Bologna criteria. Hum Reprod. 2011;26:1616- 24. PMID: 21505041 DOI: 10.1093/ humrep/der092

Gallot V, Silva AL, Genro V, Grynberg M, Frydman N, Fanchin R. Antral follicle responsiveness to follicle-stimulating hormone administration assessed by the Follicular Output RaTe (FORT) may predict in vitro fertilization-embryo transfer outcome. Hum Reprod. 2012;27:1066-72. PMID: 22279090 DOI: $10.1093 /$ humrep/der479

Genro VK, Grynberg M, Scheffer JB, Roux I, Frydman $R$, Fanchin R. Serum anti-Müllerian hormone levels are negatively related to Follicular Rate (FORT) in normocycling women undergoing controlled ovarian hyperstimulation. Hum Reprod. 2011;26:671-7. PMID: 21177311 DOI: 10.1093/humrep/deq361

Griesinger G, Teal V, McCrary Sisk C, Ruman J. Comparison of the folicular output rate after controlled ovarian stimulation with daily recombinant follicle-stimulating hormone versus Corifollitropin alfa. Eur J Obstet Gynecol Reprod Biol. 2019;232:101-5. PMID: 30529793 DOI: 10.1016/j.ejogrb.2018.11.002

Grynberg M, Labrosse J. Understanding follicular output rate (FORT) and its Implications for POSEIDON criteria. Front Endocrinol. 2019;10:246. PMID: 31040828 DOI: $10.3389 /$ fendo. 2019.00246

Kamath MS, Mascarenhas M, Franik S, Liu E, Sunkara SK. Clinical adjuncts in in vitro fertilization: a growing list. Fertil Steril. 2019;112:978-86. PMID: 31703943 DOI: 10.1016/j.fertnstert.2019.09.019

Kim $\mathrm{CH}$, Howles CM, Lee HA. The effect of transdermal testosterone gel pretreatment on controlled ovarian stimulation and IVF outcome in low responders. Fertil Steril. 2011;95:679-83. PMID: 20801436 DOI: 10.1016/j. fertnstert.2010.07.1077 
Massin N, Cedrin-Durnerin I, Coussieu C, Galey-Fontaine J, Wolf JP, Hugues JN. Effects of transdermal testosterone application on the ovarian response to $\mathrm{FSH}$ in poor responders undergoing assisted reproduction technique--a prospective, randomized, double-blind study. Hum Reprod. 2006;21:120411. PMID: 16476678 DOI: $10.1093 /$ humrep/dei481

Mazer NA. New clinical applications of transdermal testosterone delivery in men and women. J Control Release. 2000;65:30315. PMID: 10699290 DOI: 10.1016/S0168-3659(99)00252-7

Meldrum DR, Chang RJ, Giudice LC, Balasch J, Barbieri RL. Role of decreased androgens in the ovarian response to stimulation in older women. Fertil Steril. 2013;99:5-11. PMID: 23122826 DOI: 10.1016/j.fertnstert.2012.10.011

Nagels HE, Rishworth JR, Siristatidis CS, Kroon B. Androgens (dehydroepiandrosterone or testosterone) for women undergoing assisted reproduction. Cochrane Database Syst Rev. 2015;:CD009749. PMID: 26608695 DOI: $10.1002 / 14651858 . C D 009749 . p u b 2$

Polyzos NP, Davis SR, Drakopoulos P, Humaidan P, De Geyter C, Vega AG, Martinez F, Evangelou E, Van de Vijver A, Smitz J, Tournaye $\mathrm{H}$, Barri P; T-TRANSPORT Investigators Group. Testosterone for poor ovarian responders: lessons from ovarian physiology. Reprod Sci. 2018;25:980-2. PMID: 27489169 DOI: 10.1177/1933719116660849

Peñarrubia J, Fábregues F, Manau D, Creus M, Carmona F, Casamitjana R, Vanrell JA, Balasch J. Previous cycle cancelation due a to poor folicular development as a predictor of ovarian response in cycles stimulated with gonadotrophin-releasing hormone agonist-gonadotrophin treatment. Hum Reprod. 2005;20:622-8. PMID: 15608035 DOI: $10.1093 /$ humrep/deh674
Tan X, Wen Y, Chen H, Zhang L, Wang B, Wen H, Zhang F, Wu Q, Du J. Follicular output rate tends to improve clinical pregnancy outcomes in patients with polycystic ovary syndrome undergoing in vitro fertilization-embryo transfer treatment. J Int Med Res. 2019;47:5146-54. PMID: 31379237 DOI: $10.1177 / 0300060519860680$

Ubaldi F, Vaiareli A, D'Anna R, Rienzi L. Management of poor responders in IVF: is there anyting new? Biomed Res Int. 2014;2014:352098. PMID: 25136579 DOI: $10.1155 / 2014 / 352098$

Veeck LL. An atlas of human gametes and conceptuses: an illustrated reference for assisted reproductive technology. Boca Raton: CRC Press; 1999.

Vendola KA, Zhou J, Adesanya OO, Weil SJ, Bondy CA. Androgens stimulate early stages of follicular growth in the primate ovary. J Clin Invest. 1998;101:2622-9. PMID: 9637695 DOI: $10.1172 / J C I 2081$

Vendola K, Zhou J, Wang J, Famuyiwa OA, Bievre M, Bondy CA. Androgens promote oocyte insulin-like growth factor I expression and initiation of follicle development in the primate ovary. Biol Reprod. 1999;61:353-7. PMID: 10411511 DOI: $10.1095 /$ biolreprod61.2.353

Weil S, Vendola K, Zhou J, Bondy CA. Androgen and folliclestimulating hormone interactions in primate ovarian follicle development. J Clin Endocrinol Metab. 1999;84:2951-6. PMID: 10443703 DOI: $10.1210 /$ jcem.84.8.5929 